\title{
The alpha-2 receptor agonist dexmedetomidine attenuates vancomycin-induced acute kidney injury
}

\author{
Bayram A ${ }^{1}$, Erkan $\mathrm{GN}^{1}$, Talih $\mathrm{G}^{2}$, Baskol $\mathrm{G}^{3}$, Deniz $\mathrm{K}^{4}$, Yildiz $\mathrm{K}^{1}$, Esmaoglu A ${ }^{1}$ \\ Department of Anesthesiology and Reanimation, Erciyes University, Medical Faculty, Kayseri, Turkey. \\ adnanbayram1975@gmail.com
}

\section{ABSTRACT}

OBJECTIVE: This study was conducted to evaluate the protective effect of dexmedetomidine on nephrotoxicity and the mechanism of renoprotection following vancomycin-induced nephrotoxicity in rats.

METHODS: Thirty-two albino Wistar rats were divided into four groups. The control group received intraperitoneal (IP) physiological saline solution, the vancomycin (VMC) group received IP $200 \mathrm{mg} / \mathrm{kg}$ vancomycin, the dexmedetomidine (DEX) group received IP $5 \mu \mathrm{g} / \mathrm{kg}$ dexmedetomidine, and the vancomycin and dexmedetomidine (VMC+DEX) group received IP $200 \mathrm{mg} / \mathrm{kg}$ vancomycin followed by IP $5 \mu \mathrm{g} / \mathrm{kg}$ dexmedetomidine $20 \mathrm{~min}$ after the vancomycin injection. On the 8th day of the experiment, histopathological and biochemical parameters were assessed.

RESULTS: Creatinine levels were significantly higher in VMC and VMC+DEX groups. The endothelin-1 level was significantly higher in VMC group. Nitric oxide levels were statistically lower in VMC and VMC+DEX groups. Histopathologic assessments revealed that the extent of renal damage was significantly higher in group VMC ( $n=4$ with damage of Grade 3 ) compared to group VMC+DEX ( $n=0$ with damage of Grade 3 ).

CONCLUSION: It was determined that dexmedetomidine can reduce the extent of renal damage by preventing the elevation of vasoconstrictor agents (Tab. 2, Fig. 1, Ref. 36). Text in PDF www.elis.sk.

KEY WORDS: dexmedetomidine, nephrotoxicity, rat, vancomycin, vasoconstriction, vasodilation.

\section{Introduction}

Particularly due to failures in methicillin-resistant Staphylococcus aureus (MRSA) infections, higher serum levels of vancomycin are recommended to prevent bacterial resistance and to generate the treatment response $(1,2)$. However, the increased doses are strongly correlated with an increase in the risk of nephrotoxicity $(3,4)$. The incidence of vancomycin-induced nephrotoxicity (VIN) is reported to be $5-25 \%$, and even to increase to $35 \%$ when used together with aminoglycosides $(5,6)$.

Even though the VIN mechanisms have not been completely elucidated, studies have reported that free radicals and oxidative stress are involved in the process (1-8). The evidence suggesting a relationship between renal diseases and oxidative stress has led to

${ }^{1}$ Department of Anesthesiology and Reanimation, Erciyes University, Medical Faculty, Kayseri, Turkey, ${ }^{2}$ Department of Anesthesiology and Reanimation, Bozok University, Medical Faculty, Yozgat, Turkey, ${ }^{3}$ Department of Biochemistry, Erciyes University, Medical Faculty, Kayseri, Turkey, and ${ }^{4}$ Department of Pathology, Erciyes University, Medical Faculty, Kayseri, Turkey

Address for correspondence: A. Bayram, MD, Department of Anesthesiology and Reanimation, Erciyes University, Medical Faculty, Kayseri, Turkey

Phone: +90.5333609145

Acknowledgement: This study was supported by Erciyes University Scientific Research Projects Coordination Unit. ERÜ/BAP, Project No: TTU-2017-7125. the use of antioxidants in renoprotective strategies $(9,10)$. Although the use of antioxidants and agents such as erythropoietin is recommended to inhibit VIN development, it is not clear whether such applications will limit the bactericidal capacity of vancomycin(11-14).

The adrenergic receptors mediate many biological functions such as the regulation of blood flow to the organs, including the kidneys. The $\alpha_{2}$-adrenergic receptors are primarily related to the presynaptic neurons. They reduce norepinephrine secretion through their autocrine effects in the presynaptic area, thus regulating the autonomous sympathetic tonus (15). The two important mechanisms regarding the role of $\alpha_{2}$-adrenergic agonists in the protection of renal function were determined to be the reduction of sympathetic stimulation and organization of vasoreactivity (16-18).

Our hypotheses were that renal vasoconstriction might contribute to the nephrotoxic effect of vancomycin and that dexmedetomidine could inhibit vasoconstriction, thus creating the renoprotective effect. We demonstrate that our study was the first trial that investigates the protective effect of dexmedetomidine based on biochemical and histological data in the context of vancomycin-induced nephrotoxicity. Our primary purpose was to evaluate the effect of dexmedetomidine on the vancomycin-induced renal damage. Secondly, we aimed to investigate the factors that contribute to VIN.

\section{Materials and methods}

This study was conducted at the Hakan Çetinsaya Centre of Experimental and Clinical Research at Erciyes University, with 
approval from the local Ethical Committee for Animal Studies (approval number: 15/142-11.11.2015). Experimental procedures conformed to the guidelines proposed in the Guide for the Care and Use of Laboratory Animals, and to the appropriate European directives and Turkish national legislation. The study was conducted using 32 adult (10-week old) female albino Wistar rats that weighed between 170 and $230 \mathrm{~g}$. All rats were kept in standard plastic cages, under standard laboratory setting and conditions as follows: temperature of $21 \pm 2{ }^{\circ} \mathrm{C}, 55-60 \%$ humidity, and cycle of $12 \mathrm{~h}$ of light followed by $12 \mathrm{~h}$ of dark. All rats were fed with standard laboratory food and tap water, and were allowed to consume what they wanted.

\section{Experimental protocol}

The rats were randomly divided into four groups (eight per group). Each rat was weighed before the experiment to determine the required drug dosage. The four experimental groups are described below:

Control group $(\mathrm{n}=8)$ : The subjects were intraperitoneally (IP) injected with $1 \mathrm{~mL}$ physiological saline solution (PSS), and another injection of $1 \mathrm{~mL}$ PSS after $20 \mathrm{~min}$.

VMC group (vancomycin group, $\mathrm{n}=8$ ): The subjects were injected IP with $1 \mathrm{~mL}$ PSS, and 20 min later they were injected IP with $200 \mathrm{mg} / \mathrm{kg}$ vancomycin (Vankotek 1 gr vial, Koçak Farma, Turkey).

DEX group (dexmedetomidine group, $\mathrm{n}=8$ ): The subjects were injected IP with $5 \mu \mathrm{g} / \mathrm{kg}$ dexmedetomidine (Precedex 200 $\mu \mathrm{g} / 2 \mathrm{~mL}$; Hospira, Rocky Mount, NC, USA), and 20 min later they were injected IP with $1 \mathrm{~mL}$ PSS.

$\mathrm{VMC}+\mathrm{DEX}$ group (vancomycin and dexmedetomidine group, $\mathrm{n}=8$ ): The subjects were injected IP with $5 \mu \mathrm{g} / \mathrm{kg}$ dexmedetomidine, and $20 \mathrm{~min}$ later they were injected IP with $200 \mathrm{mg} / \mathrm{kg}$ vancomycin.

All injections were administered in the same volume and into the left body quadrant of the rats, while using an insulin injector by the same person. The doses were administered twice per day at 12 -h intervals. The injections were given over a period of 7 days. On the eighth day, before taking the blood samples, the rats were sedated using $50 \mathrm{mg} / \mathrm{kg}$ IP pentobarbital. Intracardiac blood samples were taken using a syringe. Both kidneys were resected by laparotomy and placed in a $10 \%$ formaldehyde solution. At the end of the experiment, the rats were sacrificed by cervical dislocation.

\section{Blood sampling and biochemical parameters in the serum}

The blood samples were centrifuged at $450 \times \mathrm{g}$ at $4{ }^{\circ} \mathrm{C}$ for 15 min. Blood urea nitrogen (BUN) and creatine $(\mathrm{Cr})$ were tested in the Swiss Roche Cobas analyzer. The serum ET-1 and NO levels were measured using the sandwich enzyme-linked immunosorbent assay (ELISA) method (using the commercial kit YL Biont, YLA0332RA, Shanghai, China). TAS (Rel Assay Kit Diagnostics RL0017, Gaziantep, Turkey) and TOS (Rel Assay Kit Diagnostics RL0024, Gaziantep, Turkey) levels were measured by spectrophotometric analysis.

\section{Histopathological assessment}

For histological examination, routine paraffin wax embedding procedures were used. The kidneys were removed, divided into sections, fixed in $10 \%$ formalin, and processed by routine histological methods. The paraffin-covered tissues were kept at room temperature until the paraffin hardened. Sections that were $5 \mu \mathrm{m}$ thick were cut from the paraffin sample blocks using a microtome (Leica RM 2155)

In order to evaluate the morphological characteristics of the tissue before assessment by light microscopy, all sections were colored with hematoxylin-eosin (H\&E) (Olympus BH-2, Olympus, Tokyo, Japan). The damage to proximal tubule cells was graded on the basis of guidance reported by Houghton and colleagues (19).

\section{Statistical analyses}

All statistical analyses were performed using SPSS 22 (SPSS, Inc., Chicago, IL, USA). Histogram and Q-Q graphs were examined and the Shapiro-Wilk test was performed to assess data normality. The Levene test was used to assess variance homogeneity. Continuous variables were presented as means with standard deviations $\left(\mathrm{SD}_{\mathrm{s}}\right)$ or medians with interquartile range (IQR). As creatinine did not show normal distribution, its results were evaluated using the Kruskal-Wallis test, while Dunn Bonferroni test was used for multiple comparisons. The values that were found to be significant were compared using pairwise comparison tests, and the statistical differences between the groups were determined. One-way analysis of variance test (ANOVA; (post hoc test) was used to compare BUN, ET-1, NO, TAS and TOS. For the groups that had differences, multiple comparisons were made using the Tukey test. A chi-squared (Fisher's exact) test was used to compare the extent of the pathological damage. The Spearman's correla-

Tab. 1. The comparison of biochemical values between groups.

\begin{tabular}{|c|c|c|c|c|}
\hline \multirow{2}{*}{ Values } & \multicolumn{4}{|c|}{ Groups } \\
\hline & Control & VMC & DEX & $\mathrm{VMC}+\mathrm{DEX}$ \\
\hline$\overline{\mathrm{BUN}}(\mathrm{mg} / \mathrm{dL})$ & $21.20 \pm 3.5^{\mathrm{a}}$ & $35.18 \pm 7.3^{\mathrm{b}}$ & $21.62 \pm 4.1^{\mathrm{a}}$ & $24.7 \pm 3.4^{\mathrm{a}}$ \\
\hline $\mathrm{Cr}(\mathrm{mg} / \mathrm{dL})$ & $0.33^{\mathrm{a}}(0.29-0.34)$ & $0.52^{\mathrm{b}}(0.38-0.54)$ & $0.33^{\mathrm{a}, \mathrm{c}}(0.32-0.35)$ & $0.40^{\mathrm{b}, \mathrm{c}}(0.38-0.42)$ \\
\hline ET-1 (ng/L) & $205.04 \pm 3.2^{\mathrm{a}}$ & $244.87 \pm 13.3^{\mathrm{b}}$ & $194.40 \pm 7.5^{\mathrm{a}}$ & $231.90 \pm 8.6^{c}$ \\
\hline $\mathrm{NO}(\mu \mathrm{mol} / \mathrm{L})$ & $219.76 \pm 31.3^{\mathrm{a}}$ & $161.48 \pm 12.9^{\mathrm{b}}$ & $242.98 \pm 38.5^{\mathrm{a}}$ & $179.25 \pm 25.8^{\mathrm{b}}$ \\
\hline TAS (mmol/ L) & $3.25 \pm 0.3$ & $3.15 \pm 0.6$ & $3.44 \pm 0.2$ & $3.42 \pm 0.3$ \\
\hline TOS $(\mu \mathrm{mol} / \mathrm{L})$ & $6.11 \pm 2.8$ & $7.22 \pm 2.8$ & $7.10 \pm 1.6$ & $7.19 \pm 2.4$ \\
\hline
\end{tabular}

BUN - blood urea nitrogen, $\mathrm{Cr}$ - creatinine, ET-1 endothelin-1, NO - nitric oxide, TAS - total antioxidant capacity, TOS - total oxidant capacity, VMC - Vancomycin group, DEX - Dexmedetomidine group, VMC+DEX - Vancomycin and dexmedetomidine group. Values are expressed as mean $\pm \mathrm{SD}$ or median (1st $-3 \mathrm{rd}$ quartiles). Same superscripts in the same row express statistically nonsignificant difference among groups. Different superscripts in the same row express statistically significant difference among groups. 
tion test was used to evaluate correlations. The limit for statistical significance was taken as $\mathrm{p}<0.01$ for the chi-squared test and $\mathrm{p}<$ 0.05 for other comparisons.

\section{Results}

\section{Biochemical assays}

The BUN value was significantly higher in VMC group compared to the other three groups $(\mathrm{p}<0.001)$ (Tab. 1). The $\mathrm{Cr}$ value was significantly higher in VMC and VMC+DEX groups compared to control group $(\mathrm{p}=0.007$ and $\mathrm{p}=0.04$, respectively). However, it was significantly lower in DEX group compared to VMC group $(\mathrm{p}=0.011)($ Tab. 1).

The ET-1 value was significantly higher in VMC group compared to the other three groups $(\mathrm{p}<0.05)$. Also, the ET-1 value of $\mathrm{VMC}+\mathrm{DEX}$ group was significantly higher than in control and DEX groups ( $p<0.05$; Table 1$)$. The NO values were significantly higher in control and DEX groups when compared to VMC and VMC+DEX groups $(\mathrm{p}<0.05)($ Tab. 1$)$.

\section{Light microscope results}

In all samples from control group, the renal damage was classified as Grade 0 (Fig. 1A). In VMC group, four kidneys were classified as Grade 2, and four kidneys were classified as Grade 3 as a result of renal damage (Fig. 1B). In DEX group, five kidneys were classified as Grade 0, and three kidneys were classified as Grade 1 (Fig. 1C). In VMC+DEX group, two kidneys were categorized as Grade 1, and six kidneys were categorized as Grade 2 (Fig. 1D). The extent of renal damage was significantly higher in VMC group ( $\mathrm{n}=4$ with Grade 3 ) compared to $\mathrm{VMC}+\mathrm{DEX}$ group ( $\mathrm{n}=0$ with Grade $3 ; \mathrm{p}<0.001$ ).

Regarding pathological damage, a strong positive correlation was observed between pathological damage and BUN or Cr. A strong negative correlation was observed between pathological damage and NO and a very strong positive correlation was observed between pathological damage and ET-1 (Tab. 2).

\section{Discussion}

The results of this study indicate that vancomycin causes renal damage in rats. It increases serum BUN, Cr and ET-1 levels, and reduces NO levels. The results also indicate that dexmedetomidine attenuates those nephrotoxic effects.

The identification of risk factors for vancomycin nephrotoxicity will help avoid serious complications (20). Ingram and co-workers evaluated 167 patients receiving vancomycin infusions (112 continuously and 55 intermittently) and concluded that the developmet of nephrotoxicity is slower in the subjects
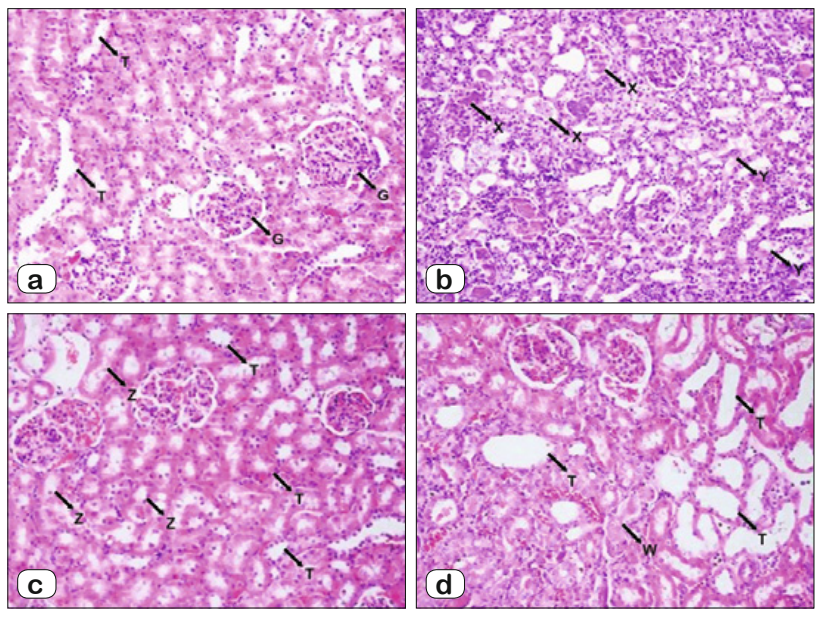

Fig. 1. Histopathological examination of rat kidney tissue. (A) Control group (H\&E × 200), (B) Vancomycin group (H\&E × 200), (C) Dexmedetomidine group (H\&E $\times 200)$, (D) Vancomycin+dexmedetomidine group (H\&E $\times$ 200) G - Glomeruli, T - Normal tubules, $X$ - Debris in damaged tubules, Y - Zones with tubular epithelial damage, W Debris in tubular lumen and focal necrosis in tubular epithelium, $\mathrm{Z}$ - Zones with minor tubular epithelial damage.

who continuously receive vancomycin infusions. However, there was no difference between the two groups regarding the prevalence (21).

Several studies have emphasized the importance of measuring the serum vancomycin concentrations for preventing nephrotoxicity in humans. Hale et al (22) indicated that there was no difference in regard to reaching the area under the curve/minimum inhibitory concentration (AUC/MIC) targets between patients with a serum vancomycin concentration of $10.0-14.9 \mathrm{mg} / \mathrm{L}$ and those with $15.0-20.0 \mathrm{mg} / \mathrm{L}$. However, they emphasized that higher serum concentrations can increase the risk of nephrotoxicity. We applied vancomycin in a dose that has been reported to be nephrotoxic, $200 \mathrm{mg} / \mathrm{kg}$, every $12 \mathrm{~h} \mathrm{(23).} \mathrm{We} \mathrm{observed} \mathrm{that} \mathrm{as} \mathrm{a} \mathrm{result} \mathrm{of} \mathrm{renal}$ damage in VMC group, four kidneys were classified as Grade 2, and four kidneys were classified as Grade 3.

It has been shown that free radicals and oxidative stress play a role in VIN, and that various antioxidant agents can prevent or reduce the damage $(11,24,25)$. Oxidative stress may set up the discharge of various vasoactive mediators and thus may straightly affect kidney functions by causing renal vasoconstriction. NO regulates the function of the blood vessels and kidneys. In animal models, the lack of NO may bring about high blood pressure with detrimental effect on vessels and kidneys. Under conditions of oxidative stress, NO is depleted and peroxynitrite increased. In this circumstance, progressive processes like vasospasm, inflam-

Tab. 2. Correlation between pathological damage and biochemical values.

\begin{tabular}{|c|c|c|c|c|c|c|}
\hline Pathological damage & BUN & $\mathrm{Cr}$ & ET-1 & $\mathrm{NO}$ & TAS & TOS \\
\hline Spearman's correlation coefficient (r) & $0,77^{*}$ & $0,77^{*}$ & $0,84^{*}$ & $-0,78^{*}$ & 0,14 & 0,07 \\
\hline $\mathrm{p}$ & $<0.001$ & $<0.001$ & $<0.001$ & $<0.001$ & 0.437 & 0.679 \\
\hline
\end{tabular}

$*<0.01$ Spearman's correlation coefficient indicates the presence of correlation 
mation, vascular and kidney disfunction may occur (26). In a review that compiled 33 randomized or retrospective observational studies, Elyasi and others determined that antioxidants such as vitamin $\mathrm{E}$, vitamin $\mathrm{C}$, and caffeic acid manifested renoprotective effects. However, they emphasized that randomized human trials would be necessary before clinical application (25). Stulak et al (27) reported that vitamin $\mathrm{C}$ and $\mathrm{E}$ supplements in pigs with hypercholesterolemia may diminish the low-density lipoprotein oxidation and increase the kidney blood flow.

Celik and co-workers investigated the possible role of free radicals in VIN, together with the effects of three different antioxidant agents and amrinone on nephrotoxicity (24). They determined that the BUN and Cr levels were significantly lower (almost two times lower) in the group that received only vancomycin compared to the group that received vancomycin together with antioxidants and amrinone. Amrinone may restrict the calcium-calmoduline complex formation that may create vasospasm via activating myosin light chain kinases and thus regulate microcirculation. Even though there was a slight increase in serum creatinine levels due to amrinone-related hypotension, no renal damage potentially leading to the necessity of renal replacement treatment was reported (28). Furthermore, it was reported that amrinone might be beneficial because it can increase renal blood flow due to its potent vasodilating effect (24).

Stockenbauer et al (29) indicated that the plasma ET-1 level increased in renal failure, and that the latter increase was correlated with the degree of renal failure. Another study indicated that NO synthesis was inhibited in the case of contrast-induced nephropathy (30). In their study on diabetic rats, Erdely et al (31) showed that in hyperglycemic obese rats, renal cortical NO synthesis had decreased, and renal damage had increased. Wang et al (32) showed that during the specific inhibition of inducible nitric oxide synthase (iNOS) and endotoxemia, the protective effect of antioxidant treatment on glomerular filtration rate (GFR) and renal blood flow had been reversed, which indicates the importance of the bioavailability of NO during early endotoxemia. Our study evaluated ET-1 and NO levels because of their roles in vasoactivity, and we found a significant positive correlation between pathological damage and ET-1, and negative correlation between pathological damage and NO levels. These findings may support the relation of vasoconstrictive mechanisms of vancomycin-induced nephrotoxicity.

There are several studies in medical literature that investigated the protective effects of dexmedetomidine on organs. Bayram et al (33) reported that dexmedetomidine had prevented an increase in plasma ET-1 and renin levels in the cases of nephropathy caused by contrast materials, which might prevent renal damage. Another study stated that dexmedetomidine suppressed sympathetic discharge by reducing presynaptic norepinephrine release, and it reduced renal damage caused by ischemia-reperfusion injury by increasing renal blood flow (34). It was also reported that dexmedetomidine had inhibited the development of long-term inflammation due to renal ischemia-reperfusion injury (35).

According to the ET-1 and NO results, the present study suggests that dexmedetomidine can have a renoprotective effect via the organization of vasoreactivity. It was also observed in this study that even though there was no significant difference between control and DEX groups, ET-1 was lower and NO was higher in the latter group. However, we suggest that the renal damage of Grade 1 observed upon histopathological examination of three kidneys in DEX group (as opposed to 0 kidneys with damage of Grade 1 in control group) might be due to hypotension caused by the dosage of dexmedetomidine used in our experiment, and subsequent development of hypoperfusion.

This study has several limitations. Firstly, we did not find any significant correlations between TAS and TOS levels and histopathological findings. We could have observed more clear results if we had studied the oxidant and antioxidant biomarkers in the kidney tissue simultaneously with serum examinations. In rats, hemodynamic measurements can be done by the telemetric method (36). Data sent through the radio-telemetric transmitter placed in the peritoneal cavity of the rat are obtained by a receiver placed under the cage. However, this study did not include telemetric hemodynamic monitoring. As a result, this study is unable to present data suggesting that the damage of Grade 1 observed in the subjects of DEX group were caused by hypotension, which can be considered as another limitation of our study.

\section{Conclusions}

The present study determined that vancomycin can cause renal damage by stimulating vasoconstrictive mechanisms and inhibiting vasodilator mediators. In contrast, dexmedetomidine can provide an opposite effect in the doses used in the study and thus can reduce the extent of renal damage. However, we would like to emphasize that dexmedetomidine can cause renal hypoperfusion secondary to hypotension. Therefore, more research is required with different doses of dexmedetomidine and close hemodynamic monitoring.

\section{References}

1. Liu C, Bayer A, Cosgrove SE et al. Clinical practice guidelines by the infectious diseases society of america for the treatment of methicillinresistant Staphylococcus aureus infections in adults and children. Clin Infect Dis 2011; 1; 52: e18-55.

2. Rybak M, Lomaestro B, Rotschafer JC et al. Therapeutic monitoring of vancomycin in adult patients: a consensus review of the American Society of Health-System Pharmacists, the Infectious Diseases Society of America, and the Society of Infectious Diseases Pharmacists. Am J Health Syst Pharm 2009; 66: 82-98.

3. Bosso JA, Nappi J, Rudisill C et al. Relationship between vancomycin trough concentrations and nephrotoxicity: a prospective multicenter trial. Antimicrob Agents Chemother 2011; 55: 5475-5479.

4. Van Hal SJ, Paterson DL, Lodise TP. Systematic review and metaanalysis of vancomycin-induced nephrotoxicity associated with dosing schedules that maintain troughs between 15 and 20 milligrams per liter. Antimicrob Agents Chemother 2013; 57: 734-744.

5. Mergenhagen KA, Borton AR. Vancomycin nephrotoxicity: a review. J Pharm Pract 2014; 27: 545-553. 
6. Elyasi S, Khalili H, Dashti-Khavidaki S, Mohammadpour A. Vancomycin-induced nephrotoxicity: mechanism, incidence, risk factors and special populations. A literature review. Eur J Clin Pharmacol 2012; 68: 1243-1255.

7. Nishino Y, Takemura S, Minamiyama Y et al. Targeting superoxide dismutase to renal proximal tubule cells attenuates vancomycin-induced nephrotoxicity in rats. Free Radic Res 2003; 37: 373-379.

8. Hazlewood KA, Brouse SD, Pitcher WD, Hall RG. Vancomycinassociated nephrotoxicity: grave concern or death by character assassination? Am J Med 2010; 123: 182 e1-7.

9. Akpinar H, Akpinar O. The effects of dexmedetomidine on biomarkers of oxidative stress and antioxidants in kidney. Bratisl Lek Listy 2018; 119: 476-480. doi: 10.4149/BLL_2018_087.

10. Tylicki L, Rutkowski B, Horl WH. Antioxidants: a possible role in kidney protection. Kidney Blood Press Res 2003; 26: 303-314.

11. Ocak S, Gorur S, Hakverdi S, Celik S, Erdogan S. Protective effects of caffeic acid phenethyl ester, vitamin $\mathrm{C}$, vitamin $\mathrm{E}$ and $\mathrm{N}$-acetylcysteine on vancomycin-induced nephrotoxicity in rats. Basic Clin Pharmacol Toxicol 2007; 100: 328-333.

12. Gupta A, Biyani M, Khaira A. Vancomycin nephrotoxicity: myths and facts. Neth J Med 2011; 69: 379-383.

13. Arimura Y, Yano T, Hirano M, Sakamoto Y, Egashira N, Oishi R. Mitochondrial superoxide production contributes to vancomycin-induced renal tubular cell apoptosis. Free Radic Biol Med 2012; 52: 1865-1873.

14. Bellomo R, Ronco C, Kellum JA, Mehta RL, Palevsky P. Acute Dialysis Quality Initiative w. Acute renal failure - definition, outcome measures, animal models, fluid therapy and information technology needs: the Second International Consensus Conference of the Acute Dialysis Quality Initiative (ADQI) Group. Crit Care 2004; 8: R204-212.

15. Wood M. Drugs and the sympathetic nervous system. In: Wood M, Wood A, eds. Drugs and Anesthesia: Pharmacology for Anesthesiologists. Baltimore, MD: Williams \& Wilkins, 1990: 375-405.

16. Kulka PJ, Tryba M, Zenz M. Preoperative alpha2-adrenergic receptor agonists prevent the deterioration of renal function after cardiac surgery: results of a randomized, controlled trial. Crit Care Med 1996; 24: 947-952.

17. Billings FTt, Chen SW, Kim M et al. alpha2-Adrenergic agonists protect against radiocontrast-induced nephropathy in mice. Am J Physiol Renal Physiol 2008; 295: F741-748.

18. Tsutsui H, Sugiura T, Hayashi K et al. Moxonidine prevents ischemia/ reperfusion-induced renal injury in rats. Eur J Pharmacol 2009; 603: 73-78.

19. Houghton DC, Plamp CE 3rd, DeFehr JM, Bennett WM, Porter G, Gilbert D. Gentamicin and tobramycin nephrotoxicity. A morphologic and functional comparison in the rat. Am J Pathol 1978; 93: 137-152.

20. da Silva NS, Muniz VD, Estofolete CF, Furtado GH, Rubio FG. Identification of temporal clusters and risk factors of bacteremia by nosocomial vancomycin-resistant enterococci. Am J Infect Control 2014; 42 : 389-392.

21. Ingram PR, Lye DC, Fisher DA, Goh WP, Tam VH. Nephrotoxicity of continuous versus intermittent infusion of vancomycin in outpatient parenteral antimicrobial therapy. Int J Antimicrob Agents 2009; 34: 570-574.
22. Hale CM, Seabury RW, Steele JM, Darko W, Miller CD. Are Vancomycin Trough Concentrations of 15 to $20 \mathrm{mg} / \mathrm{L}$ Associated With Increased Attainment of an AUC/MIC $>/=400$ in Patients With Presumed MRSA Infection? J Pharm Pract 2017; 30: 329-335.

23. Nishino Y, Takemura S, Minamiyama Y et al. Inhibition of vancomycin-induced nephrotoxicity by targeting superoxide dismutase to renal proximal tubule cells in the rat. Redox Rep 2002; 7: 317-319.

24. Celik I, Cihangiroglu M, Ilhan N, Akpolat N, Akbulut HH. Protective effects of different antioxidants and amrinone on vancomycin-induced nephrotoxicity. Basic Clin Pharmacol Toxicol 2005; 97: 325-332.

25. Elyasi S, Khalili H, Hatamkhani S, Dashti-Khavidaki S. Prevention of vancomycin induced nephrotoxicity: a review of preclinical data. Eur J Clin Pharmacol 2013; 69: 747-754.

26. Modlinger PS, Wilcox CS and Aslam S. Nitric oxide, oxidative stress, and progression of chronic renal failure. Semin Nephrol 2004; 24: 354-365.

27. Stulak JM, Lerman A, Porcel MR et al. Renal vascular function in hypercholesterolemia is preserved by chronic antioxidant supplementation. J Am Soc Nephrol.2001; 12: 1882-1891

28. Karabulut R, Sonmez K, Sancak B et al. Effects of amrinone on bilateral renal ischemia/reperfusion injury. Urol Res 2002; 30: 164-168.

29. Stockenhuber F, Gottsauner-Wolf M, Marosi L, Liebisch B, Kurz RW, Balcke P. Plasma levels of endothelin in chronic renal failure and after renal transplantation: impact on hypertension and cyclosporin A-associated nephrotoxicity. Clin Sci (Lond) 1992; 82: 255-258.

30. Agmon Y, Peleg H, Greenfeld Z, Rosen S, Brezis M. Nitric oxide and prostanoids protect the renal outer medulla from radiocontrast toxicity in the rat. J Clin Invest 1994; 94: 1069-1075.

31. Erdely A, Freshour G, Maddox DA, Olson JL, Samsell L, Baylis C. Renal disease in rats with type 2 diabetes is associated with decreased renal nitric oxide production. Diabetologia 2004; 47: 1672-1676.

32. Wang W, Jittikanont S, Falk SA et al. Interaction among nitric oxide, reactive oxygen species, and antioxidants during endotoxemia-related acute renal failure. Am J Physiol Renal Physiol 2003; 284: F532-537.

33. Bayram A, Ulgey A, Baykan A et al. The effects of dexmedetomidine on early stage renal functions in pediatric patients undergoing cardiac angiography using non-ionic contrast media: a double-blind, randomized clinical trial. Paediatr Anaesth 2014; 24: 426-432.

34. Kocoglu H, Ozturk H, Ozturk H, Yilmaz F, Gulcu N. Effect of dexmedetomidine on ischemia-reperfusion injury in rat kidney: a histopathologic study. Ren Fail 2009; 31: 70-74.

35. Si Y, Bao H, Han L et al. Dexmedetomidine protects against renal ischemia and reperfusion injury by inhibiting the JAK/STAT signaling activation. J Transl Med 2013; 11: 141.

36. Accardi MV, Troncy E, Abtout S, Ascah A, Maghezzi S, Authier S. Rat cardiovascular telemetry: Marginal distribution applied to positive control drugs. J Pharmacol Toxicol Methods 2016; 81: 120-7. 2018 by the authors. Submitted for possible open access publication under the terms and conditions of the Creative Commons Attribution (CC BY) license (http: //creativecommons.org/licenses/by/4.0/).

Received March 6, 2019. Acceted April 11, 2019. 